\title{
Main concepts in philosophy of quantum information
}

\author{
Vasil Penchev, vasildinev@gmail.com \\ Bulgarian Academy of Sciences: Institute of Philosophy and Sociology: \\ Dept. of Logical Systems and Models
}

\begin{abstract}
Quantum mechanics involves a generalized form of information, that of quantum information. It is the transfinite generalization of information and representable by transfinite ordinals. The physical world being in the current of time shares the quality of "choice". Thus quantum information can be seen as the universal substance of the world serving to describe uniformly future, past, and thus the present as the frontier of time. Future is represented as a coherent whole, present as a choice among infinitely many alternatives, and past as a well-ordering obtained as a result of a series of choices. The concept of quantum information describes the frontier of time, that "now", which transforms future into past. Quantum information generalizes information from finite to infinite series or collections. The concept of quantum information allows of any physical entity to be interpreted as some nonzero quantity of quantum information. The fundament of quantum information is the concept of 'quantum bit', "qubit". A qubit is a choice among an infinite set of alternatives. It generalizes the unit of classical information, a bit, which refer to a finite set of alternatives. The qubit is also isomorphic to a ball in Euclidean space, in which two points are chosen.
\end{abstract}

Key words: quantum information, free will, choice, qubit, quantity of quantum information, transfinite generalization of information

The thesis is: Quantum mechanics involves a generalized form of information, that of quantum information. It can be seen as the universal substance of the world and serves to describe uniformly future, past, and thus the present as the frontier of time. Quantum information generalizes information from finite to infinite series or collections.

The dimensionless physical quantity of thermodynamic entropy shares the same or similar mathematical formula as information. However it always refers to some statistical ensembles of material (energetic) entities and thus the demarcation between mass (energy) and information is conserved. Information can be considered as a quantity describing the degree of ordering (or disordering, or complexity) of any collection.

The concept of quantum information allows for any physical entity to be interpreted as some nonzero quantity of quantum information, which can be seen as that generalization of information, which is relevant to infinite collections for the classically defined information can refer only to finite ones. The quantities of mass and energy are interpretable as some nonzero amount of quantum information.

If the concept of "free will" is reduced to that of "choice" all physical world share the latter quality (Conway, Kochen 2006; 2009). Anyway the "free will" can be distinguished from the "choice": The "free will" involves implicitly a certain goal, and the choice is only the mean, by 
which the aim can be achieved or not by the one who determines the target. Thus, for example, an electron has always a choice but not free will unlike a human possessing both. Consequently, and paradoxically, the determinism of classical physics is more subjective and more anthropomorphic than the indeterminism of quantum mechanics for the former presupposes certain deterministic goal implicitly following the model of human freewill behavior.

The conception of quantum information was introduced in the theory of quantum information studying the phenomena of entanglement in quantum mechanics. The entanglement was theoretically forecast in the famous papers of Einstein, Podolsky, and Rosen (1935) and independently by Shrödinger (1935) deducing it from Hilbert space, the basic mathematical formalism of quantum mechanics. However, the former three demonstrated the forecast phenomenon as the proof of the alleged "incompleteness of quantum mechanics". John Bell (1964) deduced a sufficient condition as an experimentally verifiable criterion in order to distinguish classical from quantum correlation (entanglement). Aspect, Grangier, and Roger (1981, 1982) confirmed experimentally the existence of quantum correlations exceeding the upper limit of the possible classical correlations. The theory of quantum information has thrived since the end of the last century in the areas of quantum computer, quantum communication, and quantum cryptography.

The fundament of quantum information is the concept of 'quantum bit', "qubit" definable as the normed superposition of any two orthogonal subspaces of complex Hilbert space as follows:

'Qubit' is: $\alpha|0\rangle+\beta|1\rangle$ where $\alpha, \beta$ are complex numbers such that $|\alpha|^{2}+|\beta|^{2}=1$, and $|0\rangle,|1\rangle$ are any two orthonormal vectors (e.g. the orthonormal bases of any two subspaces) in any vector space (e.g. Hilbert space, Euclidean space, etc.). Thus Hilbert space underlying quantum mechanics is representable as the quantity of quantum information and any wave function, i.e. any state of any quantum system being a point in it can be seen as a value of that quantity. Consequently all physical processes turn out to be quantum-informational, and nature or the universe is a quantum computer processing quantum information.

The qubit is also isomorphic to a ball in Euclidean space, in which two points are chosen: A qubit is equivalently representable as a unit ball in Euclidean space and two points, the one chosen within the ball, and the other being the orthogonal projection on its surface, i.e. as a mapping of a unit ball onto its surface (or any other unit sphere).

Quantum information is equivalent to the generalization of information from finite to infinite series:

Indeed information can be interpreted as the number of choices necessary to be reached an ordering of some item from another ordering of the same item or from the absence of ordering. Then the quantity of information is the quantity of choices measured in the units of elementary choice. A bit is that unit of elementary choice: It represents the choice between two equally probable alternatives. Furthermore, the unit of quantum information, the qubit, can be interpreted as that generalization of bit, which is a choice among a continuum of alternatives. Thus it is able to measure the quantity of information as to infinite sets.

The axiom of choice is necessary for quantum information in two ways: (1) in order to guarantee the choice even if any constructive approach to be chosen an element of the continuum 
does not exist; (2) to equate the definition in terms of Hilbert space and that as a choice among a continuum of alternatives:

Indeed the theorems about the absence of hidden variables in quantum mechanics (Neumann 1932; Kochen, Specker 1968) demonstrate that the mathematical formalism of quantum mechanics implies that no well-ordering of any coherent state might exist before measurement. However, the same coherent state is transformed into a well-ordered series of results in time after measurement. In order to be equated the state before and after measurement, the well-ordering theorem equivalent to the axiom of choice is necessary. The measurement mediating between them should be interpreted as an absolutely random choice of an element of the coherent state, for which no constructive way (equivalent to some "hidden variable") can exist in principle. Thus the quantity of quantum information can describe uniformly the state before and after measurement (equivalent to a choice among an infinite set). Thus, Hilbert space can be understood as the free variable of quantum information. Then any wave function, being a given value of it, "bounds" an unorderable and a well-ordered state as the quantity of qubits (i.e. the "infinite choices") necessary for the latter to be obtained from the former.

The quantity of quantum information is the ordinal corresponding to the infinity series in question. Both definitions of 'ordinal' (Cantor 1897; Neumann 1923) are applicable as the ordinals are small. The ordinal defined in Cantor - Russell (Russell, Whitehead any edition) generates a statistical ensemble while that in Neumann, a well-ordering. Both correspond one-toone to a coherent state as the one and same quantity of quantum information containing in it.

Quantum information can be interpreted as the transfinite generalization of information. Its unit, a qubit, can be interpreted as a choice among an infinite set of alternatives. It generalizes the unit of classical information, a bit, which refer to a finite set of alternatives.

If one uses the notion of "length of now" defined as the period of the de Broglie (1925) wave associable to any physical item, the "length of now" of the apparatus can be visualized as a random chosen point onto the segment of the "length of now" of the measured quantum entity. Thus quantum mechanics is forced to describe uniformly the future, present and past of the investigated system. Future is represented as a coherent whole, present as a choice among infinitely many alternatives, and past as a well-ordering obtained as a result of a series of choices. The concept of quantum information unifies the future, present, and past as a measurable physical quantity. It describes the frontier of time, that "now", which transforms future into past. 


\section{References:}

Aspect, A., Grangier, R., Roger, G. (1981) "Experimental Tests of Realistic Local Theories via Bell's Theorem," Physical Review Letters 47 (7): 460-463.

Aspect, A., Grangier, R., Roger, G. (1982) "Experimental Realization of Einstein-Podolsky-RosenBohm Gedanken Experiment: A New Violation of Bell's Inequalities," Physical Review Letters 49 (2): 91-94.

Bell, J. (1964) "On the Einstein - Podolsky - Rosen paradox," Physics (New York) 1 (3): 195-200.

Broglie, L. de (1925) "Recherches sur la théorie des quanta (Researches on the quantum theory), [Thesis (Paris), 1924].” Annales de Physique (Paris, 10-ème série) 3: 22-128.

Cantor, G. (1897) "Beitrage zur Begrundung der transfiniten Mengenlehre (Zweiter Artikel)," Mathematische Annalen 49 (2): 207-246.

Conway, J. and Kochen, S. (2006) "The Free Will Theorem," Foundations of Physics 36 (10): 1441-1473 (arXiv:quant-ph/0604079).

Conway, J. and Kochen, S. (2009) "The Strong Free Will Theorem," Notices of the AMS 56 (2): 226232 (arXiv:0807.3286).

Einstein, A., Podolsky, B., Rosen, N. (1935) "Can Quantum-Mechanical Description of Physical Reality Be Considered Complete?” Physical Review 47 (10): 777-780.

Kochen, S., Specker, E. (1968) "The problem of hidden variables in quantum mechanics," Journal of Mathematics and Mechanics 17 (1): 59-87.

Neumann, J. von (1923) "Zur Einführung der trasfiniten Zahlen," Acta litterarum ac scientiarum Ragiae Universitatis Hungaricae Francisco-Josephinae, Sectio scientiarum mathematicarum 1 (4): 199208.

Neumann, J. von (1932). Mathematical foundation of quantum mechanics [Mathematische Grundlagen der Quantenmechanik], Berlin. Springer, pp. 167-173 (Chapter IV.2).

Schrödinger, E (1935) "Die gegenwärtige situation in der Quantenmechanik," Die Naturwissenschaften 23 (48): 807-812; 23 (49): 823-828; 23 (50): 844-849.

Whitehead, A. N., Russell, B. (any edition) Principia Mathematica, Vol. 2(*153), Vol. 3(*251). 